\title{
The natural history of asthma in childhood
}

\author{
H R ANDERSON, J M BLAND, S PATEL, AND C PECKHAM \\ From the Department of Clinical Epidemiology and Social Medicine, St George's Hospital Medical School, \\ London; and Department of Community Medicine, Institute of Child Health, London
}

SUMMARY The incidence and prognosis of childhood asthma and wheezing illness (AW) was studied using data obtained at ages 7,11, and 16 from a national cohort of 8806 children born in 1958. By the age of $16,24.7 \%$ were reported to have experienced at least one episode of AW. In $18.3 \%$ AW had started before the age of 8 , but only $4.2 \%$ continued to have symptoms in later childhood. A further $3.6 \%$ began to have $\mathrm{AW}$ between the ages of 8 and 11 , and $2.8 \%$ began between the ages of 12 and 16 . Of those with AW at age 7, 28.3\% had symptoms at 11 and $16.5 \%$ at 16 ; these proportions were about doubled if AW at 7 had been severe. The associations between natural history and a large number of perinatal, social, environmental, and medical factors were examined. Those which predicted the onset of AW after the age of 7 were: male sex of child; mother aged 15-19 at child's birth; history of pneumonia, whooping cough, throat or ear infections or tonsillectomy; eczema, allergic rhinitis; and periodic vomiting or abdominal pain.

To understand the natural history of asthma, a description of both its incidence and prognosis is required. Prevalence rates obtained by cross-sectional population studies reflect only the net effect of incidence and prognosis, and those relying on retrospective assessments have the additional problem of inadequate and selective recall. With one exception ${ }^{1}$ previously reported cohort studies have been essentially follow up studies designed only to study prognosis since only subjects with asthma were seen subsequently. ${ }^{2-5}$

The National Child Development Study, ${ }^{6}$ which originated in the National Perinatal Study, ${ }^{7}$ has followed up at ages 7,11, and 16 nearly every child in England, Scotland, and Wales born in one week of March 1958. Data, which were collected by parent interview and physical examination of the child, comprised a wide variety of perinatal, medical, social, behavioural, and educational variables. Since the study was not designed to investigate the natural history of asthma there were, not unexpectedly, shortcomings in the assessment of both asthma and the range of aetiological factors. Nevertheless the size and representativeness of this national cohort offer a unique opportunity to examine the natural history of asthma and wheezing illness.

\section{Methods}

Information about current and past asthma or wheezing was obtained as part of a structured questionnaire on medical and other topics administered to parents by health visitors. Data for all three interviews (at ages 7, 11, and 16) were obtained for 8806 of the original cohort of 17419 births comprising the 1958 Births Survey $(98 \%$ of all births in that week). Most of the analysis in this paper relates to the 8806 children with complete data, but, as we shall show, there was no evidence of a difference in asthma and wheeze between these children and those for whom data were incomplete.

The form of the questions on asthma or wheezing differed at each age. At age 7, parents were asked whether their child had ever had "attacks of asthma," and the same question was repeated for "attacks of bronchitis with wheezing"; if positive to either question, the number of attacks in the past 12 months was recorded. This latter information is not available on the existing data archive and needed to be extracted especially for the present study from the microfiche records. At age 11, the parent was asked whether the child had ever had attacks of "asthma," "wheezing bronchitis," or neither of these. If attacks had occurred in the past 12 months, their frequency was assessed as follows: "at least once a week, usually less than once a week but can expect one a month, at least one attack in the past year but less frequently than once a month, had attacks in past year but don't know how frequently, no attacks in past year but had attacks when younger". At age 16 the parent was asked whether the child "ever had an attack of asthma or wheezy bronchitis". If attacks had occurred in the past 
122

12 months frequency was categorised as: "at least once a week, usually less than once a week but at least once a month, less than once a month, frequency unknown."

Since at age 16 the question did not distinguish between asthma and wheezing and because there are no good clinical or pathological grounds for distinguishing them, ${ }^{2}$ these two entities were combined at ages 7 and 11 and will be referred to as asthma or wheezing (AW). Thus, at each age there were three possibilities: no AW, AW but not in the past 12 months, and current AW (in past 12 months). Using the reports of frequency of attacks in the past 12 months, a further division according to severity was
$H$ R Anderson, J M Bland, S Patel and C Peckham possible: five or more attacks in the last year (at age 7) and attacks occurring at least once a month (at 11 and 16) being classified as "severe." To examine the natural history of $\mathrm{AW}$, subjects were assigned to one of 27 mutually exclusive categories on the basis of their response to the question about asthma or wheezing illness at each interview (table 1). Taking into account the numbers in each category and their potential clinical or epidemiological usefulness, these were collapsed into six main categories: (1) AW before the age of 7 but not current at 7 or thereafter; (2) AW at age 7 but not thereafter; (3) AW at or before age 7 and at 11 or 16 ; (4) first appearance of $\mathrm{AW}$ between

Table 1 Asthma or wheezy bronchitis reported at each interview. Range of possible responses grouped into natural history categories

\begin{tabular}{|c|c|c|c|c|c|c|c|c|}
\hline \multirow{3}{*}{$\begin{array}{l}\text { Natural history } \\
\text { category }\end{array}$} & \multicolumn{8}{|c|}{ Report at each interview } \\
\hline & \multicolumn{2}{|c|}{ Age 7 years } & \multicolumn{2}{|c|}{ Age 11 years } & \multicolumn{2}{|c|}{ Age 16 years } & \multirow[b]{2}{*}{ No. } & \multirow{2}{*}{$\begin{array}{l}\text { Percentage } \\
\text { of total }\end{array}$} \\
\hline & Ever & Last year & Ever & Last year & Ever & Last year & & \\
\hline Never & No & No & No & No & No & No & 6630 & $75 \cdot 3$ \\
\hline By 7 years and not after & $\begin{array}{l}\text { Yes } \\
\text { Yes } \\
\text { Yes } \\
\text { Yes }\end{array}$ & $\begin{array}{l}\text { No } \\
\text { No } \\
\text { No } \\
\text { No }\end{array}$ & $\begin{array}{l}\text { No } \\
\text { No } \\
\text { Yes* } \\
\text { Yes* }\end{array}$ & $\begin{array}{l}\text { No } \\
\text { No } \\
\text { No } \\
\text { No }\end{array}$ & $\begin{array}{l}\text { No } \\
\text { Yes* } \\
\text { No } \\
\text { Yes* }\end{array}$ & $\begin{array}{l}\text { No } \\
\text { No } \\
\text { No } \\
\text { No }\end{array}$ & $\begin{array}{r}452 \\
71 \\
112 \\
123\end{array}$ & $\begin{array}{l}5.1 \\
0.8 \\
1.3 \\
1.4\end{array}$ \\
\hline Subtotal & & & & & & & 758 & $8 \cdot 6$ \\
\hline At 7 years and not after & $\begin{array}{l}\text { Yes } \\
\text { Yes } \\
\text { Yes } \\
\text { Yes }\end{array}$ & $\begin{array}{l}\text { Yes } \\
\text { Yes } \\
\text { Yes } \\
\text { Yes }\end{array}$ & $\begin{array}{l}\text { No } \\
\text { No } \\
\text { Yes* } \\
\text { Yes* }\end{array}$ & $\begin{array}{l}\text { No } \\
\text { No } \\
\text { No } \\
\text { No }\end{array}$ & $\begin{array}{l}\text { No } \\
\text { Yes* } \\
\text { No } \\
\text { Yes* }\end{array}$ & $\begin{array}{l}\text { No } \\
\text { No } \\
\text { No } \\
\text { No }\end{array}$ & $\begin{array}{r}268 \\
36 \\
77 \\
101\end{array}$ & $\begin{array}{l}3.0 \\
0.4 \\
0.9 \\
1.1\end{array}$ \\
\hline Subtotal & & & & & & & 482 & $5 \cdot 5$ \\
\hline 0-7 years and after & $\begin{array}{l}\text { Yes } \\
\text { Yes } \\
\text { Yes } \\
\text { Yes } \\
\text { Yes } \\
\text { Yes } \\
\text { Yes } \\
\text { Yes } \\
\text { Yes } \\
\text { Yes }\end{array}$ & $\begin{array}{l}\text { No } \\
\text { No } \\
\text { No } \\
\text { No } \\
\text { No } \\
\text { Yes } \\
\text { Yes } \\
\text { Yes } \\
\text { Yes } \\
\text { Yes }\end{array}$ & $\begin{array}{l}\text { No } \\
\text { Yes } \\
\text { Yes } \\
\text { Yes } \\
\text { Yes } \\
\text { No } \\
\text { Yes } \\
\text { Yes } \\
\text { Yes } \\
\text { Yes }\end{array}$ & $\begin{array}{l}\text { No } \\
\text { No } \\
\text { Yes } \\
\text { Yes } \\
\text { Yes } \\
\text { No } \\
\text { No } \\
\text { Yes } \\
\text { Yes } \\
\text { Yes }\end{array}$ & $\begin{array}{l}\text { Yes } \\
\text { Yes } \\
\text { No } \\
\text { Yes } \\
\text { Yes } \\
\text { Yes } \\
\text { Yes } \\
\text { No } \\
\text { Yes } \\
\text { Yes }\end{array}$ & $\begin{array}{l}\text { Yes } \\
\text { Yes } \\
\text { No } \\
\text { No } \\
\text { Yes } \\
\text { Yes } \\
\text { Yes } \\
\text { No } \\
\text { No } \\
\text { Yes }\end{array}$ & $\begin{array}{l}20 \\
11 \\
17 \\
50 \\
21 \\
18 \\
24 \\
39 \\
91 \\
77\end{array}$ & $\begin{array}{l}0.2 \\
0.1 \\
0.2 \\
0.6 \\
0.2 \\
0.2 \\
0.3 \\
0.4 \\
1.0 \\
0.9\end{array}$ \\
\hline Subtotal & & & & & & & 368 & $4 \cdot 2$ \\
\hline 8-11 years onset & $\begin{array}{l}\text { No } \\
\text { No } \\
\text { No } \\
\text { No } \\
\text { No } \\
\text { No }\end{array}$ & $\begin{array}{l}\text { No } \\
\text { No } \\
\text { No } \\
\text { No } \\
\text { No } \\
\text { No }\end{array}$ & $\begin{array}{l}\text { Yes } \\
\text { Yes } \\
\text { Yes } \\
\text { Yes } \\
\text { Yes } \\
\text { Yes }\end{array}$ & $\begin{array}{l}\text { No } \\
\text { No } \\
\text { Yes } \\
\text { Yes } \\
\text { No } \\
\text { Yes }\end{array}$ & $\begin{array}{l}\text { No } \\
\text { Yes } \\
\text { No } \\
\text { Yes } \\
\text { Yes } \\
\text { Yes }\end{array}$ & $\begin{array}{l}\text { No } \\
\text { No } \\
\text { No } \\
\text { No } \\
\text { Yes } \\
\text { Yes }\end{array}$ & $\begin{array}{r}136 \\
56 \\
56 \\
36 \\
8 \\
27\end{array}$ & $\begin{array}{l}1.5 \\
0.6 \\
0.6 \\
0.4 \\
0.1 \\
0.3\end{array}$ \\
\hline Subtotal & & & & & & & 319 & 3.6 \\
\hline $12-16$ years onset & $\begin{array}{l}\text { No } \\
\text { No }\end{array}$ & $\begin{array}{l}\text { No } \\
\text { No }\end{array}$ & $\begin{array}{l}\text { No } \\
\text { No }\end{array}$ & $\begin{array}{l}\text { No } \\
\text { No }\end{array}$ & $\begin{array}{l}\text { Yes } \\
\text { Yes }\end{array}$ & $\begin{array}{l}\text { No } \\
\text { Yes }\end{array}$ & $\begin{array}{l}149 \\
100\end{array}$ & $\begin{array}{l}1 \cdot 7 \\
1 \cdot 1\end{array}$ \\
\hline Subtotal & & & & & & & 249 & 2.8 \\
\hline Total & & & & & & & 8806 & 100 \\
\hline
\end{tabular}

"In these subjects the time of disappearance of AW is not clear; they have therefore been assigned to the earliest age band in which the occurrence of symptoms was certain. 
interviews at age 7 and age 11 ; (5) first appearance of AW between interviews at age 11 and 16; (6) no report of AW at any interview.

In addition to the interview, other relevant data were available from the Perinatal Survey (extracted from obstetric records) and from the school doctor's examination made at each age. From the very large number of variables possible we selected all those that had ever, to our knowledge, been associated even speculatively with the natural history of asthma. The overall association between each variable and the six natural history categories was examined using the chi squared test or one-way analysis of variance, as appropriate. For those showing a statistically significant association the relative risks of each natural history category occurring were calculated; this was done by dividing the incidence of a particular category in those who had the factor by the incidence in those who had not. The $95 \%$ confidence intervals for the relative risks were then calculated.

\section{Results}

\section{INCIDENCE, PROGNOSIS, AND PREVALENCE}

The various combinations of questionnaire response are shown in table 1 . This method of presentation enables calculation of both incidence and prognosis, as well as a 12-month period prevalence and cumulative life time prevalence. A total of $24.7 \%$ of the cohort had experienced AW at some time in their lives; $8.6 \%$ had experienced transient AW in early life, disappearing before age $7,5 \cdot 5 \%$ had current asthma at age 7 but not thereafter, $3.6 \%$ started their asthma between the ages of 8 and $11,2.8 \%$ began their AW between 12 and 16 , and $4.2 \%$ had AW throughout childhood.

When interviewed at age 16 the reported life time prevalence was $11.6 \%$ whereas the life time prevalence by 16 obtained using data from all three interviews was $24.7 \%$ (table 2). The prevalence of current asthma was highest at age $7(8.3 \%)$ followed by $4.7 \%$ at 11 and $3.5 \%$ at 16 . The rates for severe (frequent) current asthma were much less, being $0.89 \%, 0.64 \%$, and $0.5 \%$ respectively. The cumulative life time prevalence of severe asthma was $1.8 \%$. Table 2 also shows that the prevalence of $\mathrm{AW}$ in children whose parents were interviewed on all three occasions was similar to that among those seen only once or twice, indicating that rates are unlikely to be biased by response.

Prognosis was related to severity at age 7. Of those with any current $\mathrm{AW}$ at $7,28 \cdot 3 \%$ reported symptoms at $11,16.3 \%$ reported symptoms at 16 , and $10.5 \%$ reported symptoms at both 11 and 16 . If the AW was severe at age 7, the proportion with symptoms at 11 was $64.1 \%$ (16.7\% severe), at $1635.9 \%$ (3.9\% severe), and at both 11 and 16 was $28 \cdot 2 \%$ (2.6\% severe).
Table 2 Prevalence of asthma or wheezy bronchitis $(A W)$ at each interview

\begin{tabular}{|c|c|c|c|}
\hline & \multicolumn{3}{|c|}{ Age at interview (years) } \\
\hline & 7 & 11 & 16 \\
\hline \multicolumn{4}{|l|}{$\begin{array}{l}\text { Prevalence of a past history of } \\
\text { AW at each interview }\end{array}$} \\
\hline $\begin{array}{l}\text { Linked data } \\
\text { (information at all interviews) }\end{array}$ & $\begin{array}{l}18 \cdot 3 \% \\
1608 / 8806\end{array}$ & $\begin{array}{l}12 \cdot 1 \% \\
1062 / 8806\end{array}$ & $\begin{array}{l}11 \cdot 6 \% \\
1019 / 8806\end{array}$ \\
\hline $\begin{array}{l}\text { Unlinked data } \\
\text { (no information at one or more } \\
\text { interviews) }\end{array}$ & $\begin{array}{l}18 \cdot 3 \% \\
1057 / 5765\end{array}$ & $\begin{array}{l}12 \cdot 7 \% \\
601 / 4751\end{array}$ & $\begin{array}{l}11 \cdot 8 \% \\
308 / 2610\end{array}$ \\
\hline All interviewed & $\begin{array}{l}18 \cdot 3 \% \\
2665 / 14571\end{array}$ & $\begin{array}{l}12 \cdot 3 \% \\
1663 / 13557\end{array}$ & $\begin{array}{l}11 \cdot 6 \\
1327 / 11416\end{array}$ \\
\hline $\begin{array}{l}\text { Cumulative lifetime prevalence } \\
\text { up to } 16 \text { years (linked data) }\end{array}$ & $\begin{array}{l}18 \cdot 3 \% \\
1608 / 8806\end{array}$ & $\begin{array}{l}21 \cdot 9 \% \\
1927 / 8806\end{array}$ & $\begin{array}{l}24 \cdot 7 \% \\
2176 / 8806\end{array}$ \\
\hline \multicolumn{4}{|l|}{$\begin{array}{l}\text { Prevalence of AW in past } 12 \\
\text { months, at each interview }\end{array}$} \\
\hline Linked data & $\begin{array}{l}8 \cdot 3 \% \\
731 / 8806\end{array}$ & $\begin{array}{l}4 \cdot 7 \% \\
414 / 8806\end{array}$ & $\begin{array}{l}3 \cdot 5 \% \\
306 / 8806\end{array}$ \\
\hline Unlinked data & $\begin{array}{l}7.8 \% \\
450 / 5765\end{array}$ & $\begin{array}{l}5.3 \% \\
250 / 4751\end{array}$ & $\begin{array}{l}3.9 \% \\
101 / 2610\end{array}$ \\
\hline All interviewed & $\begin{array}{l}8 \cdot 1 \% \\
1181 / 14571\end{array}$ & $\begin{array}{l}4.9 \% \\
664 / 13557\end{array}$ & $\begin{array}{l}3.6 \% \\
407 / 11416\end{array}$ \\
\hline $\begin{array}{l}\text { Prevalence of frequent AW at each } \\
\text { interview in linked data }\end{array}$ & $\begin{array}{l}0.89 \% \\
78 / 8806\end{array}$ & $\begin{array}{l}0.64 \% \\
56 / 8806\end{array}$ & $\begin{array}{l}0.5 \% \\
44 / 8806\end{array}$ \\
\hline \multirow{2}{*}{$\begin{array}{l}\text { Cumulative prevalence of frequent } \\
\text { AW based on } 12 \text { month period } \\
\text { prevalences at } 7,11 \text {, and } 16 \text { years }\end{array}$} & & & \\
\hline & $\begin{array}{l}0.89 \% \\
78 / 8806\end{array}$ & $\begin{array}{l}1.4 \% \\
121 / 8806\end{array}$ & $\begin{array}{l}1.8 \% \\
155 / 8806\end{array}$ \\
\hline
\end{tabular}

BIOLOGICAL, SOCIAL, AND ENVIRONMENTAL FACTORS

Of the large number of such factors considered, few were found to be associated with the pattern of natural history of AW, and these are shown in table 3. Factors that were not found to be related are described in the text. Boys were more likely than girls to have continuing symptoms or to develop AW in later childhood, but the sexes were equally likely to have symptoms appearing in early life but disappearing after age 7.

The most prominent perinatal factor was the age of the mother at the birth of the child, those born to the youngest mothers having a higher risk of all categories of natural history. There was no association with birthweight, gestational age, parity, breast feeding, birth order or rank in the family. Smoking during pregnancy was associated only with a higher risk of transient $\mathrm{AW}$ before the age of 7. Multifactorial analysis confirmed that although mother's age was related to both social class and breast feeding, neither of these factors could explain its relation to AW.

The sharing of household amenities (bathroom, toilet) at the time of interview (at ages 7 and 11) was associated with an increased risk of AW at age 7 but not thereafter. No associations were found with crowding, number of children in the household, tenure 
Table 3 Biological, social, and environmental variables associated with natural history of asthma or wheezy bronchitis

\begin{tabular}{|c|c|c|c|c|c|c|c|c|}
\hline \multirow{3}{*}{$\frac{\text { Variable }}{\text { Sex of child }}$} & \multirow{3}{*}{$\begin{array}{l}\begin{array}{l}\text { Overall } x^{2} \\
\text { probability }\end{array} \\
<0.001\end{array}$} & \multirow[b]{2}{*}{$\begin{array}{l}\text { Relative risk of: } \\
\text { (95\% confidence } \\
\text { interval) }\end{array}$} & \multicolumn{6}{|c|}{ Natural history category } \\
\hline & & & \multirow{2}{*}{$\begin{array}{l}\begin{array}{l}\text { Never } \\
n=6630\end{array} \\
0.9 \\
(0.8-1 \cdot 0)\end{array}$} & \multirow{2}{*}{$\begin{array}{l}\text { By } 7 \text { yr } \\
\text { and not after } \\
n=758\end{array}$} & \multirow{2}{*}{$\begin{array}{l}\text { At } 7 \mathrm{yr} \\
\text { and not after } \\
n=482\end{array}$} & \multirow{2}{*}{$\begin{array}{l}0-7 y r \\
\text { and after } \\
n=368\end{array}$} & \multirow{2}{*}{$\begin{array}{l}\begin{array}{l}8-11 \mathrm{yr} \\
\text { onset } \\
n=319\end{array} \\
\begin{array}{l}1 \cdot 3^{*} \\
(1 \cdot 0-1 \cdot 6)\end{array}\end{array}$} & \multirow{2}{*}{$\begin{array}{l}\begin{array}{l}12-16 y r \\
\text { onset } \\
n=249\end{array} \\
\begin{array}{l}1 \cdot 4^{*} \\
(1 \cdot 1-1 \cdot 8)\end{array}\end{array}$} \\
\hline & & Boy: & & & & & & \\
\hline $\begin{array}{l}\text { Mother's age at } \\
\text { child's birth }\end{array}$ & $<0.001$ & $\begin{array}{ll}15-19: & 20-29 \mathrm{yr} \\
15-19: & 30+\mathrm{yr} \\
20-29: & 30+\mathrm{yr}\end{array}$ & $\begin{array}{l}0.9 \\
(0.7-1 \cdot 0) \\
0.8 \\
(0.7-1 \cdot 0) \\
1.0 \\
(0.9-1 \cdot 1)\end{array}$ & $\begin{array}{l}1.4^{*} \\
(1.0-1 \cdot 4) \\
1.6^{*} \\
(1.2-2.2) \\
1.2 \\
(1.0-1.4)\end{array}$ & $\begin{array}{l}1.5^{*} \\
(1.0-2.2) \\
1.3 \\
(0.9-1.9) \\
0.9 \\
(0.7-1 \cdot 1)\end{array}$ & $\begin{array}{l}1 \cdot 1 \\
(0 \cdot 7-1 \cdot 8) \\
1 \cdot 3 \\
(0 \cdot 8-2 \cdot 1) \\
1 \cdot 1 \\
(0 \cdot 9-1 \cdot 4)\end{array}$ & $\begin{array}{l}1.9^{*} \\
(1 \cdot 2-2.8) \\
1.9 * \\
(1.2-3.0) \\
1.0 \\
(0.8-1 \cdot 3)\end{array}$ & $\begin{array}{l}1.7^{*} \\
(1.0-2.7) \\
2.0 * \\
(1.2-3.4) \\
1.2 \\
(0.9-1.6)\end{array}$ \\
\hline $\begin{array}{l}\text { Smoking after } 4 \text { th } \\
\text { month of pregnancy }\end{array}$ & $<0.001$ & $\begin{array}{l}\text { Smoker: } \\
\text { Non-Smoker }\end{array}$ & $\begin{array}{l}1.0 \\
(0.9-1 \cdot 1)\end{array}$ & $\begin{array}{l}1 \cdot 3^{*} \\
(1 \cdot 1-1 \cdot 5)\end{array}$ & $\begin{array}{l}1 \cdot 2 \\
(1 \cdot 0-1 \cdot 4)\end{array}$ & $\begin{array}{l}0.8 \\
(0.6-1 \cdot 0)\end{array}$ & $\begin{array}{l}1 \cdot 0 \\
(0 \cdot 8-1 \cdot 2)\end{array}$ & $\begin{array}{l}1.0 \\
(0.8-1 \cdot 3)\end{array}$ \\
\hline Region of child's birth & $<<0.010$ & $\begin{array}{ll}\text { North: } & \text { Centre } \\
\text { North: } & \text { South } \\
\text { Centre: South }\end{array}$ & $\begin{array}{l}1 \cdot 1 \\
1 \cdot 1 \\
1 \cdot 0\end{array}$ & $\begin{array}{l}0.7^{*} \\
0.8^{*} \\
1.1\end{array}$ & $\begin{array}{l}0.9 \\
0.9 \\
1.0\end{array}$ & $\begin{array}{l}0.9 \\
1.0 \\
1.0\end{array}$ & $\begin{array}{l}0.7 \\
0.9 \\
1.2\end{array}$ & $\begin{array}{l}1.0 \\
1.0 \\
1.0\end{array}$ \\
\hline $\begin{array}{c}\text { Household facilities } \\
\text { at age } 7\end{array}$ & $<0.008$ & $\begin{array}{l}\text { At least one } \\
\text { shared: none } \\
\text { shared }\end{array}$ & $\begin{array}{l}1 \cdot 0 \\
(0 \cdot 9-1 \cdot 1)\end{array}$ & $\begin{array}{l}1 \cdot 1 \\
(0 \cdot 9-1 \cdot 3)\end{array}$ & $\begin{array}{l}1 \cdot 5^{*} \\
(1 \cdot 2-1 \cdot 8)\end{array}$ & $\begin{array}{l}0.9 \\
(0.7-1.2)\end{array}$ & $\begin{array}{l}1 \cdot 0 \\
(0 \cdot 7-1 \cdot 3)\end{array}$ & $\begin{array}{l}0.8 \\
(0.6-1 \cdot 2)\end{array}$ \\
\hline at age 11 & $<0.050$ & $\begin{array}{l}\text { At least one } \\
\text { shared: none } \\
\text { none }\end{array}$ & $\begin{array}{l}1 \cdot 0 \\
(0.8-1 \cdot 1)\end{array}$ & $\begin{array}{l}1.0 \\
(0 \cdot 8-1 \cdot 2)\end{array}$ & $\begin{array}{l}1 \cdot 4^{*} \\
(1 \cdot 1-1 \cdot 6)\end{array}$ & $\begin{array}{l}1 \cdot 1 \\
(0 \cdot 8-1 \cdot 6)\end{array}$ & $\begin{array}{l}0.8 \\
(0.6-1 \cdot 2)\end{array}$ & $\begin{array}{l}1 \cdot 1 \\
(0.8-1 \cdot 7)\end{array}$ \\
\hline
\end{tabular}

${ }^{*} \mathrm{p}<0.05$

of accommodation or social class (whether assessed at birth, age 7 or 11). Separation from the mother had no effect, even after analysing by age at separation and length of separation. There was no association with being in care or with the absence of one or both biological parents from the household.

To look for geographical effects, three geographical areas, North (Scotland and Northern Region), Centre (East and West Ridings, North Western Wales, Midlands and North Midlands), and South (South Western, Southern, Eastern, London and South Eastern) were defined. A tendency was observed for children in the North to experience less transient AW before age 7 than in the rest of the country, but no other regional effect was observed.

\section{RESPIRATORY ILLNESSES}

The associations of upper and lower respiratory illness are shown in table 4. A history of pneumonia at the 7 year interview was not only associated with $\mathrm{AW}$ during the first 7 years but with onset between 12 and 16 years (relative risk $=1 \cdot 75$ ). Further analysis found no evidence of an association between the age at which the first attack of pneumonia occurred and subsequent risk of AW. A history of whooping cough was associated with an increased risk of all categories of natural history. There was no association with a history of measles-asked at ages 7 and 11. Upper respiratory illnesses affecting the throat and ears were associated with increased risk of concurrent natural history categories but, with the exception of "running ears" at age 11, did not predict the later onset of $\mathrm{AW}$. Removal of tonsils or adenoids reported at age 7 was associated with a small increase in risk for all categories of natural history. This was significant for those with transient $\mathrm{AW}$ before age 7, and onset between 12 and 16 . Those who reported this operation at 11 showed significant associations only with increased risk of $\mathrm{AW}$ in the first 7 years and not thereafter. Analysis by age at operation (asked at age 7) showed no effect.

\section{ECZEMA AND ALLERGIC RHINITIS}

The relation of $A W$ to eczema, and hayfever or allergic rhinitis, is shown in table 5. Eczema was assessed not only by interview but at ages 7 and 11 , by medical examination. However assessed, the findings were consistent with a more than four-fold increase in relative risk for those experiencing $\mathrm{AW}$ throughout childhood but with no significant risk for AW which disappeared after the age of 7. Eczema before or at 7 also predicted the later onset of AW. The association with hayfever or allergic rhinitis was very similar in pattern and degree to that with eczema. Those children who had a history at age 7 of both eczema after the first year and of hayfever/allergic rhinitis showed a 
Table 4 Past and present medical factors associated with natural history of asthma or wheezy bronchitis

\begin{tabular}{|c|c|c|c|c|c|c|c|c|c|}
\hline \multirow{3}{*}{$\begin{array}{l}\text { Variable } \\
\begin{array}{l}\text { Pneumonia ever } \\
(7 \mathrm{yr})\end{array}\end{array}$} & \multirow{3}{*}{$\begin{array}{c}\begin{array}{c}\text { Overall } x^{2} \\
\text { probability }\end{array} \\
<0.001\end{array}$} & & & \multicolumn{6}{|c|}{ Natural history category } \\
\hline & & \multicolumn{2}{|c|}{$\begin{array}{l}\text { Relative risk of: } \\
\text { (95\% confidence } \\
\text { interval) }\end{array}$} & \multirow{2}{*}{$\begin{array}{l}\begin{array}{l}\text { Never } \\
n=6630\end{array} \\
\begin{array}{l}0.6^{*} \\
(0.5-0.8)\end{array}\end{array}$} & \multirow{2}{*}{$\begin{array}{l}\begin{array}{l}B y \\
\text { and not } \\
\text { after } \\
n=758\end{array} \\
\begin{array}{l}2 \cdot 0^{*} \\
(1 \cdot 5-2 \cdot 7)\end{array}\end{array}$} & \multirow{2}{*}{$\begin{array}{l}\text { At } 7 y r \\
\text { and not after } \\
n=482\end{array}$} & \multirow{2}{*}{$\begin{array}{l}\begin{array}{l}0-7 y r \\
\text { and after } \\
n=368\end{array} \\
4 \cdot 3^{*} \\
(3 \cdot 2-5 \cdot 8)\end{array}$} & \multirow{2}{*}{$\begin{array}{l}\begin{array}{l}8-11 \text { yr } \\
\text { onset } \\
n=319\end{array} \\
\begin{array}{l}1.5 \\
(1 \cdot 0-2 \cdot 4)\end{array}\end{array}$} & \multirow{2}{*}{$\begin{array}{l}\begin{array}{l}12-16 y r \\
\text { onset } \\
n=249\end{array} \\
\begin{array}{l}1 \cdot 8^{*} \\
(1 \cdot 1-2 \cdot 9)\end{array}\end{array}$} \\
\hline & & Yes: & No & & & & & & \\
\hline $\begin{array}{l}\text { Whooping cough ever } \\
(7 \mathrm{yr})\end{array}$ & $<0.001$ & Yes: & No & $\begin{array}{l}0.9 \\
(0.8-1.0)\end{array}$ & $\begin{array}{l}1 \cdot 4^{*} \\
(1 \cdot 2-1 \cdot 7)\end{array}$ & $\begin{array}{l}1.2 \\
(0.9-1 \cdot 5)\end{array}$ & $\begin{array}{l}1 \cdot 4^{*} \\
(1 \cdot 1-1 \cdot 8)\end{array}$ & $\begin{array}{l}1.2 \\
(0.9-1.6)\end{array}$ & $\begin{array}{l}1.3 \\
(0.9-1.8)\end{array}$ \\
\hline $\begin{array}{l}\text { Whooping cough ever } \\
\text { (11 yr) }\end{array}$ & $<0.001$ & Yes: & No & $\begin{array}{l}0.9 \\
(0.3-1.0)\end{array}$ & $\begin{array}{l}1.2^{*} \\
(1.0-1 \cdot 5)\end{array}$ & $\begin{array}{l}1 \cdot 3^{*} \\
(1 \cdot 0-1 \cdot 6)\end{array}$ & $\begin{array}{l}1 \cdot 4^{*} \\
(1 \cdot 1-1 \cdot 7)\end{array}$ & $\begin{array}{l}1 \cdot 4^{*} \\
(1 \cdot 1-1 \cdot 8)\end{array}$ & $\begin{array}{l}1.4^{*} \\
(1.0-1.8)\end{array}$ \\
\hline $\begin{array}{l}\text { Throat and/or ear } \\
\text { infections with fever: } \\
\text { More than } 3 \text { in past } \\
\text { year }(7 \mathrm{yr})\end{array}$ & $<0.001$ & Yes: & No & $\begin{array}{l}0.9 \\
(0.8-1 \cdot 1)\end{array}$ & $\begin{array}{l}1 \cdot 2 \\
(1 \cdot 0-1 \cdot 5)\end{array}$ & $\begin{array}{l}1 \cdot 6^{*} \\
(1 \cdot 3-2 \cdot 0)\end{array}$ & $\begin{array}{l}1 \cdot 4^{*} \\
(1 \cdot 0-1 \cdot 8)\end{array}$ & $\begin{array}{l}0.7 \\
(0.5-1 \cdot 1)\end{array}$ & $\begin{array}{l}1 \cdot 0 \\
(0.7-1 \cdot 5)\end{array}$ \\
\hline $\begin{array}{l}\text { Throat and/or ear } \\
\text { infections treated by } \\
\text { doctor-Recurrent in } \\
\text { past year (11 yr) }\end{array}$ & $<0.001$ & Yes: & No & $\begin{array}{l}0.9 \\
(0.8-1 \cdot 1)\end{array}$ & $\begin{array}{l}1.1 \\
(0.9-1.4)\end{array}$ & $\begin{array}{l}1.0 \\
(0.8-1.4)\end{array}$ & $\begin{array}{l}1 \cdot 5^{*} \\
(1 \cdot 1-2 \cdot 0)\end{array}$ & $\begin{array}{l}1 \cdot 7 * \\
(1 \cdot 3-2 \cdot 3)\end{array}$ & $\begin{array}{l}1 \cdot 1 \\
(0.8-1 \cdot 6)\end{array}$ \\
\hline $\begin{array}{l}\text { Running ears ever } \\
(7 \mathrm{yr})\end{array}$ & $<0.032$ & Yes: & No & $\begin{array}{l}0.9 \\
(0.8-1 \cdot 1)\end{array}$ & $\begin{array}{l}1 \cdot 3^{*} \\
(1 \cdot 1-1 \cdot 7)\end{array}$ & $\begin{array}{l}1 \cdot 3 \\
(0 \cdot 9-1 \cdot 7)\end{array}$ & $\begin{array}{l}0.9 \\
(0 \cdot 6-1 \cdot 3)\end{array}$ & $\begin{array}{l}1 \cdot 0 \\
(0 \cdot 7-1 \cdot 5)\end{array}$ & $\begin{array}{l}1.2 \\
(0.8-1.9)\end{array}$ \\
\hline $\begin{array}{l}\text { Discharging ears in } \\
\text { past year (11 yr) }\end{array}$ & $<0.075$ & Yes: & No & $\begin{array}{l}0.9 \\
(0.7-1 \cdot 2)\end{array}$ & $\begin{array}{l}1.2 \\
(0.8-1.9)\end{array}$ & $\begin{array}{l}1 \cdot 3 \\
(0 \cdot 8-2 \cdot 3)\end{array}$ & $\begin{array}{l}1 \cdot 8^{*} \\
(1 \cdot 0-3 \cdot 0)\end{array}$ & $\begin{array}{l}1.6 \\
(0.9-2.9)\end{array}$ & $\begin{array}{l}0.7 \\
(0.3-2 \cdot 0)\end{array}$ \\
\hline $\begin{array}{l}\text { Tonsils and/or } \\
\text { adenoids removed } \\
\text { ( } 7 \mathrm{yr})\end{array}$ & $<0.001$ & Yes: & No & $\begin{array}{l}0.9 \\
(0.8-1 \cdot 1)\end{array}$ & $\begin{array}{l}1 \cdot 3^{*} \\
(1 \cdot 0-1 \cdot 5)\end{array}$ & $\begin{array}{l}1 \cdot 2 \\
(1 \cdot 0-1 \cdot 5)\end{array}$ & $\begin{array}{l}1 \cdot 2 \\
(0.9-1 \cdot 6)\end{array}$ & $\begin{array}{l}1.2 \\
(0.9-1.6)\end{array}$ & $\begin{array}{l}1.4^{*} \\
(1.0-1.9)\end{array}$ \\
\hline $\begin{array}{l}\text { Tonsils and/or } \\
\text { adenoids removed } \\
\text { (11 yr) }\end{array}$ & $<0.001$ & Yes: & No & $\begin{array}{l}0.9 \\
(0.8-1 \cdot 1)\end{array}$ & $\begin{array}{l}1 \cdot 2^{*} \\
(1 \cdot 0-1 \cdot 4)\end{array}$ & $\begin{array}{l}1 \cdot 3^{*} \\
(1 \cdot 1-1 \cdot 6)\end{array}$ & $\begin{array}{l}1.2 \\
(0.9-1 \cdot 5)\end{array}$ & $\begin{array}{l}1 \cdot 2 \\
(1 \cdot 0-1 \cdot 7)\end{array}$ & $\begin{array}{l}1.0 \\
(0.8-1.4)\end{array}$ \\
\hline $\begin{array}{l}\text { Fit or convulsion- } \\
\text { in first year of life } \\
(7 \mathrm{yr})\end{array}$ & $<0.001$ & Yes: & No & $\begin{array}{l}0.9 \\
(0.6-1 \cdot 2)\end{array}$ & $\begin{array}{l}1.2 \\
(0.7-2 \cdot 0)\end{array}$ & $\begin{array}{l}1 \cdot 8^{*} \\
(1 \cdot 1-3 \cdot 0)\end{array}$ & $\begin{array}{l}2 \cdot 7^{*} \\
(1 \cdot 6-4 \cdot 4)\end{array}$ & $\begin{array}{l}1 \cdot 0 \\
(0 \cdot 4-2 \cdot 3)\end{array}$ & $\begin{array}{l}0.6 \\
(0 \cdot 2-2 \cdot 0)\end{array}$ \\
\hline $\begin{array}{l}\text { Wet by day after } \\
3 \text { years }(7 \mathrm{yr})\end{array}$ & $<0.004$ & Yes: & No & $\begin{array}{l}0.9 \\
(0.7-1 \cdot 1)\end{array}$ & $\begin{array}{l}1 \cdot 2 \\
(0 \cdot 4-1 \cdot 7)\end{array}$ & $\begin{array}{l}1 \cdot 7 * \\
(1 \cdot 2-2 \cdot 5)\end{array}$ & $\begin{array}{l}1.0 \\
(0 \cdot 6-1 \cdot 6)\end{array}$ & $\begin{array}{l}1.5 \\
(1.0-2 \cdot 4)\end{array}$ & $\begin{array}{l}1 \cdot 2 \\
(0 \cdot 6-2 \cdot 1)\end{array}$ \\
\hline $\begin{array}{l}\text { Wet by night after } \\
5 \text { years }(7 \mathrm{yr})\end{array}$ & $<0.001$ & Yes: & No & $\begin{array}{l}0.9 \\
(0.8-1 \cdot 1)\end{array}$ & $\begin{array}{l}1.5^{*} \\
(1.2-1.9)\end{array}$ & $\begin{array}{l}1.2 \\
(0.9-1.6)\end{array}$ & $\begin{array}{l}1.0 \\
(0.7-1.4)\end{array}$ & $\begin{array}{l}1.2 \\
(0.8-1 \cdot 6)\end{array}$ & $\begin{array}{l}1 \cdot 1 \\
(0.7-1 \cdot 6)\end{array}$ \\
\hline
\end{tabular}

$* p<0.05$

remarkably low relative risk of never having AW $(R=0.36)$ and a remarkably high risk of having AW throughout childhood $(R=15 \cdot 1)$.

HEADACHES, VOMITING, AND ABDOMINAL PAIN (table 5)

Headaches or migraine at age 7 were associated with AW over that period of life and with AW which continued into later childhood; the relative risk of $\mathrm{AW}$ starting between 12 and 16 was increased but fell short of significance $(R=1 \cdot 39,95 \%$ CI $0.9-2 \cdot 1)$. Headaches at age 11 were associated only with an increase in the risk of chronic AW (reported at each interview).

Both recurrent vomiting and recurrent abdominal pain reported at age 7 were associated with an increased risk of all natural history categories except onset between 8 and 11. Reports of these symptoms at 11 were not associated with current or subsequent AW.

\section{MISCELLANEOUS}

Children reported to have had fits in the first year of life were more likely to have had AW currently at age 7 and throughout childhood; however, those whose fits continued past the first year of life showed no increase in the risk of any category of AW. Enuresis was associated only with an increased risk of AW in early life, but not after 7 years of age.

There were no associations with age at menarche, pubic hair rating of boys, current smoking by parents (at 16) or current smoking by child (at 16). 
Table 5 Association between eczema, hayfever, abdominal pain, vomiting, and headaches with natural history of asthma or wheezy bronchitis

\begin{tabular}{|c|c|c|c|c|c|c|c|c|c|c|}
\hline \multirow{3}{*}{$\begin{array}{l}\text { Variable } \\
\text { Eczema in first year } \\
(7 \mathrm{yr})\end{array}$} & \multirow{3}{*}{$\begin{array}{c}\begin{array}{c}\text { Overall } x^{2} \\
\text { probability }\end{array} \\
<0.001\end{array}$} & \multirow{2}{*}{\multicolumn{2}{|c|}{$\begin{array}{l}\text { Relative risk of: } \\
\text { (95\% confidence } \\
\text { interval) }\end{array}$}} & \multicolumn{6}{|c|}{ Natural history category } & \\
\hline & & & & \multirow{2}{*}{$\begin{array}{l}\begin{array}{l}\text { Never } \\
n=6630\end{array} \\
\begin{array}{l}0.7^{*} \\
(0.6-0.9)\end{array}\end{array}$} & \multirow{2}{*}{$\begin{array}{l}\text { By } 7 y r \\
\text { and not after } \\
n=758\end{array}$} & \multirow{2}{*}{$\begin{array}{l}\text { At } 7 \mathrm{yr} \\
\text { and not after } \\
n=482\end{array}$} & \multirow{2}{*}{ 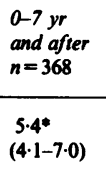 } & \multirow{2}{*}{$\begin{array}{l}\begin{array}{l}8-11 y r \\
\text { onset } \\
n=319\end{array} \\
\begin{array}{l}1 \cdot 7 * \\
(1 \cdot 1-2 \cdot 6)\end{array}\end{array}$} & \multirow{2}{*}{$\begin{array}{l}\begin{array}{l}12-16 y r \\
\text { onset } \\
n=249\end{array} \\
\begin{array}{c}1.5 \\
(0.9-2.5)\end{array}\end{array}$} & \\
\hline & & Yes: & No & & & & & & & \\
\hline $\begin{array}{l}\text { Eczema after first } \\
\text { year }(7 \mathrm{yr})\end{array}$ & $<0.001$ & Yes: & No & $\begin{array}{l}0.8^{*} \\
(0.6-0.9)\end{array}$ & $\begin{array}{l}1.1 \\
(0.8-1.5)\end{array}$ & $\begin{array}{l}1.3 \\
(0.9-1.7)\end{array}$ & $\begin{array}{l}4 \cdot 7 * \\
(3 \cdot 6-6 \cdot 2)\end{array}$ & $\begin{array}{l}1 \cdot 3 \\
(0 \cdot 8-2 \cdot 0)\end{array}$ & $\begin{array}{l}1 \cdot 7^{*} \\
(1 \cdot 1-2 \cdot 6)\end{array}$ & \\
\hline $\begin{array}{l}\text { Eczema on } \\
\text { examination by doctor } \\
(7 \mathrm{yr})\end{array}$ & $<0.001$ & Yes: & No & $\begin{array}{l}0.7^{*} \\
(0.6-1 \cdot 0)\end{array}$ & $\begin{array}{l}0.8 \\
(0.5-1 \cdot 4)\end{array}$ & $\begin{array}{l}1 \cdot 1 \\
(0.7-2 \cdot 0)\end{array}$ & $\begin{array}{l}4 \cdot 9 * \\
(3 \cdot 5-7 \cdot 0)\end{array}$ & $\begin{array}{l}1.6 \\
(0.9-2.9)\end{array}$ & $\begin{array}{l}2 \cdot 1^{*} \\
(1 \cdot 2-3 \cdot 8)\end{array}$ & \\
\hline $\begin{array}{l}\text { Eczema on } \\
\text { examination by doctor } \\
\text { (11 yr) }\end{array}$ & $<0.001$ & Yes: & No & $\begin{array}{l}0.7^{*} \\
(0.5-0.9)\end{array}$ & $\begin{array}{l}1.3 \\
(0.8-2 \cdot 0)\end{array}$ & $\begin{array}{l}1.4 \\
(0.8-2 \cdot 3)\end{array}$ & $\begin{array}{l}4 \cdot 3^{*} \\
(2 \cdot 9-6 \cdot 4)\end{array}$ & $\begin{array}{l}2 \cdot 5^{*} \\
(1 \cdot 5-4 \cdot 4)\end{array}$ & $\begin{array}{l}1.7 \\
(0.9-3 \cdot 3)\end{array}$ & \\
\hline $\begin{array}{l}\text { Eczema in past year } \\
(11 \mathrm{yr})\end{array}$ & $<0.001$ & Yes: & No & $\begin{array}{l}0.7^{*} \\
(0.6-0.9)\end{array}$ & $\begin{array}{l}1.2 \\
(0.8-1 \cdot 7)\end{array}$ & $\begin{array}{l}1.2 \\
(0.8-1.9)\end{array}$ & $\begin{array}{l}4 \cdot 2^{*} \\
(3 \cdot 1-5 \cdot 7)\end{array}$ & $\begin{array}{l}1.9^{*} \\
(1 \cdot 3-3 \cdot 0)\end{array}$ & $\begin{array}{l}1 \cdot 7^{*} \\
(1 \cdot 0-2 \cdot 8)\end{array}$ & \\
\hline $\begin{array}{l}\text { Hayfever or sneezing } \\
\text { attacks ever (7 yr) }\end{array}$ & $<0.001$ & Yes: & No & $\begin{array}{l}0.6^{*} \\
(0.5-0.7)\end{array}$ & $\begin{array}{l}1 \cdot 3 \\
(1 \cdot 0-1 \cdot 7)\end{array}$ & $\begin{array}{l}2 \cdot 0^{*} \\
(1 \cdot 5-2 \cdot 7)\end{array}$ & $\begin{array}{l}7 \cdot 1^{*} \\
(5 \cdot 6-9 \cdot 1)\end{array}$ & $\begin{array}{l}1.5 \\
(1 \cdot 0-2 \cdot 2)\end{array}$ & $\begin{array}{l}1 \cdot 7 * \\
(1 \cdot 1-2 \cdot 7)\end{array}$ & \\
\hline $\begin{array}{l}\text { Hayfever or allergic } \\
\text { rhinitis in past year } \\
\text { (11 yr) }\end{array}$ & $<0.001$ & Yes: & No & $\begin{array}{l}0.7 * \\
(0.6-0.9)\end{array}$ & $\begin{array}{l}1 \cdot 0 \\
(0.7-1 \cdot 3)\end{array}$ & $\begin{array}{l}1 \cdot 2 \\
(0 \cdot 9-1 \cdot 6)\end{array}$ & $\begin{array}{l}5 \cdot 2^{*} \\
(4 \cdot 1-6 \cdot 6)\end{array}$ & $\begin{array}{l}2 \cdot 2^{*} \\
(1 \cdot 6-3 \cdot 1)\end{array}$ & $\begin{array}{l}1.9 * \\
(1 \cdot 3-2 \cdot 7)\end{array}$ & \\
\hline $\begin{array}{l}\text { Eczema after first year } \\
\text { plus history of } \\
\text { hayfever (both asked } \\
\text { at } 7 \mathrm{yr} \text { ) }\end{array}$ & $<0.001$ & Yes: & Neither & $\begin{array}{l}0.4^{*} \\
(0.2-0.6)\end{array}$ & $\begin{array}{l}1 \cdot 2 \\
(0 \cdot 6-2 \cdot 4)\end{array}$ & $\begin{array}{l}1 \cdot 6 \\
(0.8-3 \cdot 3)\end{array}$ & $\begin{array}{l}15 \cdot 1^{*} \\
(9 \cdot 9-23 \cdot 0)\end{array}$ & $\begin{array}{l}1.8 \\
(0.8-4 \cdot 1)\end{array}$ & $\begin{array}{l}2.0 \\
(0.8-4.9)\end{array}$ & రి \\
\hline $\begin{array}{l}\text { Frequent headaches or } \\
\text { migraine ever }(7 \mathrm{yr})\end{array}$ & $<0.001$ & Yes: & No & $\begin{array}{l}0.9 \\
(0.7-1.0)\end{array}$ & $\begin{array}{l}1 \cdot 3 * \\
(1 \cdot 1-1 \cdot 7)\end{array}$ & $\begin{array}{l}1 \cdot 5^{*} \\
(1 \cdot 1-1 \cdot 9)\end{array}$ & $\begin{array}{l}2 \cdot 2^{*} \\
(1 \cdot 6-2 \cdot 9)\end{array}$ & $\begin{array}{l}0.8 \\
(0.6-1 \cdot 3)\end{array}$ & $\begin{array}{l}1 \cdot 4 \\
(0 \cdot 9-2 \cdot 1)\end{array}$ & 它 \\
\hline $\begin{array}{l}\text { Recurrent headaches } \\
\text { or migraine in past } \\
\text { year (11 yr) }\end{array}$ & $<0.001$ & Yes: & No & $\begin{array}{l}0.9 \\
(0.8-1 \cdot 1)\end{array}$ & $\begin{array}{l}1 \cdot 2 \\
(1 \cdot 0-1 \cdot 5)\end{array}$ & $\begin{array}{l}1 \cdot 1 \\
(0.9-1 \cdot 4)\end{array}$ & $\begin{array}{l}1 \cdot 6^{*} \\
(1 \cdot 2-2 \cdot 0)\end{array}$ & $\begin{array}{l}1 \cdot 2 \\
(0.9-1 \cdot 7)\end{array}$ & $\begin{array}{l}1 \cdot 1 \\
(0.8-1 \cdot 6)\end{array}$ & \\
\hline $\begin{array}{l}\text { Periodic vomiting or } \\
\text { bilious attacks ever } \\
\text { ( } 7 \mathrm{yr} \text { ) }\end{array}$ & $<0.001$ & Yes: & No & $\begin{array}{l}0.9 \\
(0.8-1.0)\end{array}$ & $\begin{array}{l}1.2 * \\
(1.0-1 \cdot 5)\end{array}$ & $\begin{array}{l}1 \cdot 4^{*} \\
(1 \cdot 2-1 \cdot 8)\end{array}$ & $\begin{array}{l}1 \cdot 8^{*} \\
(1 \cdot 4-2 \cdot 3)\end{array}$ & $\begin{array}{l}0.8 \\
(0 \cdot 6-1 \cdot 1)\end{array}$ & $\begin{array}{l}1.4^{*} \\
(1 \cdot 0-1 \cdot 9)\end{array}$ & \\
\hline $\begin{array}{l}\text { Recurrent vomiting } \\
\text { or bilious attacks } \\
\text { in past year (11 yr) }\end{array}$ & $<0.091$ & Yes: & No & $\begin{array}{l}0.9 \\
(0.7-1.2)\end{array}$ & $\begin{array}{l}1.0 \\
(0.7-1.4)\end{array}$ & $\begin{array}{l}1 \cdot 5^{*} \\
(1 \cdot 0-2 \cdot 2)\end{array}$ & $\begin{array}{l}1 \cdot 3 \\
(0 \cdot 8-2 \cdot 0)\end{array}$ & $\begin{array}{l}1 \cdot 5 \\
(0 \cdot 9-2 \cdot 3)\end{array}$ & $\begin{array}{l}1 \cdot 0 \\
(0.6-1 \cdot 9)\end{array}$ & \\
\hline $\begin{array}{l}\text { Periodic abdominal } \\
\text { pain ever (7 yr) }\end{array}$ & $<0.001$ & Yes: & No & $\begin{array}{l}0.9 \\
(0.8-1 \cdot 3)\end{array}$ & $\begin{array}{l}1 \cdot 4^{*} \\
(1 \cdot 1-1 \cdot 7)\end{array}$ & $\begin{array}{l}1 \cdot 3^{*} \\
(1 \cdot 1-1 \cdot 7)\end{array}$ & $\begin{array}{l}1 \cdot 5 * \\
(1 \cdot 1-1 \cdot 9)\end{array}$ & $\begin{array}{l}0.9 \\
(0.6-1 \cdot 2)\end{array}$ & $\begin{array}{l}1.4^{*} \\
(1.0-1.9)\end{array}$ & \\
\hline $\begin{array}{l}\text { Recurrent abdominal } \\
\text { pain in past year } \\
(11 \mathrm{yr})\end{array}$ & $<0.032$ & Yes: & No & $\begin{array}{l}1 \cdot 0 \\
(0.8-1 \cdot 1)\end{array}$ & $\begin{array}{l}1 \cdot 3^{*} \\
(1 \cdot 1-1 \cdot 7)\end{array}$ & $\begin{array}{l}1.0 \\
(0.8-1.4)\end{array}$ & $\begin{array}{l}1.1 \\
(0.8-1.5)\end{array}$ & $\begin{array}{l}1 \cdot 3 \\
(0.9-1 \cdot 7)\end{array}$ & $\begin{array}{l}0.8 \\
(0.5-1.3)\end{array}$ & \\
\hline
\end{tabular}

\section{Discussion}

Before discussing the implications of the findings several methodological aspects need to be considered. The first concerns the possibility of bias due to incomplete follow up. Response has been analysed and reported in considerable detail. ${ }^{89}$ At each individual sweep, the response has been satisfactory: at birth, $98 \%$; at 7 years, $91 \%$; at 11 years, $91 \%$; and at
16 years, 87\%. However, the proportions with parental interview data on which the present analysis is based were lower- $69 \%$ for the sweep at age 16 . No response bias was found for birthweight, indices of physical growth, a history of asthma or the occupation, education or attitudes of the parents. The main response bias was that children with indicators of "disadvantage" were under represented, but this did not exceed $10 \%$ for any particular index. We did not 
find that, at any age, the prevalence rates among children on whom we had linked data were materially different from those not seen on each occasion (table 2). We therefore conclude that it is unlikely that response bias has had any important effect on our estimates of incidence, prognosis or relative risk. Secondly, as the NCDS was not set up primarily to examine the natural history of $\mathrm{AW}$, it was not possible to assess certain aetiologically important factors nor to assess all those examined fully. For example, the parents' smoking habits were not assessed at either age 7 or 11 , nor is there information on parental atopic disorders. Thirdly, the identification of AW differed at different ages of interview as to phrasing of the questions and to the method of grading frequency. This is unlikely to have influenced the results to any great extent because both diagnosed asthma and wheezing illness were assessed at each interview, as was their occurrence over the past 12 months, which is the conventional period for describing "current" asthma. Indeed, the prevalence estimates at age 7 were similar to those observed in some other population surveysin which all wheezingillness was included. ${ }^{2}$ 10-12

The only other study adequately designed to examine the natural history of wheezing in a population based cohort was reported by Giles et al from Tasmania. This study obtained linked data on 7132 children assessed at ages 7 and 13, and on 658 subjects assessed at 7,13 , and 20 (following stratified sampling). The Tasmanian study is stronger than ours in respect of its more detailed and consistent assessment of respiratory symptoms and lung function. On the other hand, the present study has followed a larger number of subjects and examined a wider variety of possible aetiological factors.

Reports of prognosis in the literature are expressed in a variety of ways, making comparisons difficult. For this reason we have presented data in table 1 so as to allow others to calculate whatever incidence or prognosis they may require. The importance of taking severity into account in calculating prognosis is clearly important but difficult for comparative purposes because there is no agreement about the assessment of severity in chronic asthma. Nevertheless, our findings in relation to prognosis are in agreement with those of McNicol and Williams ${ }^{2}$ who, in their population based survey of Melbourne schoolchildren, found that of children with any current wheezing at age 7 only $30 \%$ still had symptoms at 10 and $25 \%$ at 14 . Their figure of $19.1 \%$ for the life time prevalence of wheeze reported at age 11 is also close to our finding of $21.9 \%$. The finding that about half of those with severe asthma at age 7 still have symptoms at 16 is similar to that from follow-ups of clinical cases. ${ }^{1314}$ The Tasmanian study ${ }^{1}$ estimated that $3.6 \%$ of all their cohort wheezed at each assessment, that is, up to age
20. This is remarkably close to our figure of $4.6 \%$ for those wheezing throughout childhood. It is difficult to make further comparisons of prognosis with this study.

The incidence of AW throughout childhood reached $24.7 \%$, which resembles estimates made by Fry $^{15}$ and Goodall ${ }^{16}$ in their respective general practices. A considerable proportion (6.5\%) developed AW between 8 and 16 years. However, the prevalence at 16 was only $3.5 \%$, and this amply demonstrates that a much higher proportion of children experience AW for some time in their childhood than would be suggested by cross sectional studies. The lack of reliability of retrospective reports of $\mathrm{AW}$ is also demonstrated. Giles $e^{t}$ al $^{1}$ estimated an annual incidence of about $0.6 \%$ between the ages of 7 and 20; this compares closely with our own result that $6.4 \%$ developed asthma between age 7 and 16, an average annual incidence of $0.7 \%$ per annum. In addition, their estimate of incidence up to age 7 $(16.2 \%)$ is remarkably similar to that of the present study $(18 \cdot 3 \%)$.

From an aetiological viewpoint the various factors found to be associated with natural history can be divided into two: those concurrent with $\mathrm{AW}$ and those which preceded the AW. For those factors which were concurrently associated with $\mathrm{AW}$, and not shown to precede the onset of $\mathrm{AW}$, the problem of making causal inferences is the same as for a cross sectional or case-control study in that it may be difficult to distinguish cause and effect. For example, the association between fits in the first year of life and AW beginning before age 7 might be explained by a febrile episode associated with a chest infection itself predisposed to by asthma or the asthmatic tendency. Concurrent associations with AW were observed for household amenities, throat and/or ear infections, enuresis, and frequent headaches. The importance of the present study lies, however, in the second type of association, that in which a particular factor of interest was assessed before the onset of $\mathrm{AW}$ and could therefore be considered as a potential risk factor. The tables have concentrated on the positive findings but the negative findings listed in the text are also of considerable importance for those factors where there has been speculation or conflict in the literature.

Our results confirm the almost universal finding that boys are at greater risk than girls and show that this is most important in those with chronic asthma and with later onset; the latter observation conflicts with some prevalence studies which have indicated a decline in male to female ratio as puberty approaches. ${ }^{17}$

Among the perinatal factors, the most important and, to our knowledge, new finding was that the risk of all natural history categories apart from persistent AW was increased in children whose mothers were 
under 20 at the time of their birth. This was significant not only for AW up to 7, but for onset from 8 years onwards. This effect could not be explained by the relation between mothers' age at the birth and social class or breast feeding. Parity, birth order, gestational age, and birthweight had no effect.

The absence of an association with breast feeding does not agree with the findings of Blair, ${ }^{13}$ who found that asthma was more likely to persist in those who were bottle fed. Smoking during pregnancy was associated with transient $\mathrm{AW}$ before age 7 . A plausible explanation for this may be that these mothers smoked subsequent to the pregnancy and that the respiratory symptoms were the effect of passive smoking. ${ }^{18-21}$ If this is so, it is reassuring to note that maternal smoking had no effect on subsequent onset of asthma or chronic asthma. This is consistent with the finding that parents' smoking at 16 was not associated with an increased risk of AW.

The regional analysis showed that those in the Northern part of Great Britain (Scotland and Northern Region) had a lower risk of transient AW before age 7 and there were also lower, though not significant, risks for the other natural history categories. This could represent a differing response to the questionnaire but is nevertheless consistent with existing data which show a lower prevalence in the more Northern latitudes of Europe. ${ }^{17}$

Most population surveys have found that family stress is not an important correlate of asthma. ${ }^{22}{ }^{23} \mathrm{Our}$ results are in agreement since a wide range of factors indicative of family stress, including separation from the mother, lack of one or more natural parents in the home, being in care, and crowding, had no effect.

There was no effect of social class or housing tenure, which agrees with some though not all prevalence surveys. Using the present cohort, a previous report of a social class relationship described an association between social class and asthma at age $7^{6}$ and at 11 but not with wheezy bronchitis. ${ }^{24}$ The disappearance of this association when all wheezing illness is considered suggests that it represents a class effect on the illness label rather than on the disease itself. This lack of association with social class or housing tenure is important because these are the commonly used indicators of the range of life styles and living conditions within Great Britain. Thus the environmental factor causing $\mathrm{AW}$ appears to be operating at a different level.

Recurrent headaches were associated with $\mathrm{AW}$ as were recurrent abdominal pain or vomiting attacks, which also significantly predicted the later onset of AW. Associations of AW with both of these symptoms have been observed in prevalence surveys. ${ }^{23} 24$ Understanding the significance of these associations might throw new light on the mechanism of asthma.
Another new and potentially important finding was that a history of pneumonia (and of whooping cough) increased the risk of all natural history categories, including adolescent onset. Two studies, one prospective $^{25}$ and the other retrospective, ${ }^{26}$ have linked respiratory disease in adults to that in early childhood. A number of shorter term prospective studies have also shown that early respiratory problems are associated with symptoms in later childhood, 192728 and reduced ventilatory capacity. ${ }^{1927}$ One of these ${ }^{27}$ showed that non-wheezing respiratory illness (pneumonia, bronchitis) was associated with wheeze at a later age but did not report continuing and new cases separately. An increased risk of wheeze in children with a history of whooping cough has also been reported. ${ }^{27}$

One explanation for these associations is that some of the reports of pneumonia in our study were referring to what was in fact asthma. But if the history of pneumonia is accepted at face value as a non-asthmatic lower respiratory tract infection, the following theories can be constructed to explain itso prediction of the onset of asthma. The first is tha pneumonia caused the airways to become hyperreactive, ie, caused the onset of the asthmatice tendency. ${ }^{26}$ If this was the case, it is difficult to explaing why such a long symptom-free period followed the pneumonia for those whose onset was in adolescence $\frac{O}{\oplus}$ A second explanation is that both pneumonia and $\overrightarrow{0} . \overrightarrow{0}$ wheeze might have a common environmental cause $\frac{0}{5}$ such as passive smoking. Since we did not find an effect of smoking of parents at age 16, this seems unlikely. A third theory is that both pneumonia and the AW reflect a predisposition to chest disease in general. This is supported by recent evidence that the increased tendency to wheeze among children with previous whooping cough precedes the attack of whooping cough and cannot therefore have been caused by it. ${ }^{29} \mathrm{~A}$ variant of this hypothesis is that the asthmatic tendency itself is the underlying factor and is responsible not only for wheezing illness but increases the risk of other lower respiratory tract illnesses. ${ }^{30}$

We observed, as did others, ${ }^{131}$ that current smoking of the child at age 16 was equally common in those with and without AW. Asthmatics who continue to smoke are likely, however, to accelerate the decline of their lung function and may be at greater risk of chronic airflow obstruction in adult life.

We thank' Dr K Fogelman and his colleagues at the National Children's Bureau and the ESRC Data Archive for giving us access to the data, and Nick Tait for help with the analysis. 


\section{References}

${ }^{1}$ Giles GG, Lickiss N, Gibson HB, Shaw K. Respiratory symptoms in Tasmanian adolescents: a follow up of the 1961 birth cohort. Aust NZ J Med 1984; 14: 631-7.

2 Williams H, McNicol KN. Prevalence, natural history, and relationship of wheezy bronchitis and asthma in children. An epidemiological study. Br Med J 1969; 4: 321-5.

${ }^{3}$ McNicol KN, Williams HB. Spectrum of asthma in children-I, clinical and physiological components. $\mathrm{Br}$ Med J 1973; 4: 7-11.

${ }^{4}$ Martin AJ, McLennan LA, Landau LI, Phelan PD. The natural history of childhood asthma to adult life. $\mathrm{Br} \mathrm{Med}$ $J$ 1980; 280: 1397-1400.

${ }^{5}$ Lancet. Long-term prognosis in asthma. Lancet 1977; 2: 1015.

${ }^{6}$ Davie R, Butler N, Goldstein H. From birth to seven. The Second Report of the National Child Development Study (1958 cohort). London: National Children's Bureau, 1972.

${ }^{7}$ Butler NR, Alberman E (eds). Perinatal problems. London: Livingstone, 1969.

${ }^{8}$ Fogelman K (ed). Britain's sixteen-year olds. London: National Children's Bureau, 1976.

${ }^{9}$ Fogelman K (ed). Growing up in Great Britain: Papers from the National Child Development Study London: National Children's Bureau Series, 1983.

${ }^{10}$ Burr ML, Eldridge BA, Borysiewicz LK. Peak expiratory flow rates before and after exercise in schoolchildren. Arch Dis Child 1974; 49: 923-6.

11 Anderson HR, Bailey PA, Cooper JS, Palmer JC, West S. Morbidity and school absence caused by asthma and wheezing illness. Arch Dis Child 1983; 58: 777-84.

12 Speight ÂN, Lee DA, Hey EN. Underdiagnosis and undertreatment of asthma in childhood. Br Med J 1983; 286: $1253-6$.

13 Blair H. Natural history of childhood asthma. 20-year follow-up. Arch Dis Child 1977; 52: 613-9.

14 Rackemann FM, Edwards MC. Asthma in children. A follow-up study of 688 patients after an interval of twenty years. $N$ Engl J Med 1952; 246: 815-23.

15 Fry J. "Acute Wheezy Chests." Clinical patterns and natural history. Br Med J 1961; 1: 227-32.

16 Goodall JF. The natural history of common respiratory infection in children and some principles in its management III. Wheezy children. $J R$ Coll Gen Pract 1958; 1: 51-9.
${ }^{17}$ Gregg I. Epidemiological aspects. In: Clark TJH, Godfrey $\mathrm{S}$ (eds). Asthma, 2nd edition. London: Chapman and Hall Medical, 1983; 242.

${ }^{18}$ Charlton A. Children's coughs related to parental smoking. Br Med J 1984; 288: 1647-9.

${ }^{19}$ Leeder SR, Corkhill RT, Wysocki MJ, Holland WW, Colley JRT. Influence of personal and family factors on ventilatory function of children. Br J Prev Soc Med 1976; 30: 219-24.

${ }^{20}$ Liard R, Perdrizet S, Reinert P. Wheezy bronchitis in infants and parents' smoking habits. Lancet 1982; 1: $334-5$.

${ }^{21}$ Colley JRT, Holland WW, Corkhill RT. Influence of passive smoking and parental phlegm on pneumonia and bronchitis in early childhood. Lancet 1974; 2: 1031-4.

${ }^{22}$ McNicol KN, Williams HE, Allan J, McAndrew I. Spectrum of asthma in children-III, Psychological and social components. $\mathrm{Br} \mathrm{Med} J$ 1973; 4: 16-20.

${ }^{23}$ Anderson HR, Bailey PA, Cooper JS, Palmer JC, West S Morbidity and school absence caused by asthma and wheezing illness. Arch Dis Child 1983; 58: 777-84.

${ }^{24}$ Peckham C, Butler N. A national study of asthma in childhood. J Epidemiol Community Health 1978; 32: 79-85.

${ }^{25}$ Colley JRT, Douglas JWB, Reid DD. Respiratory disease in young adults: influence of early childhood lower respiratory tract illness, social class, air pollution, and smoking. Br Med J 1973; ii: 195-8.

${ }^{26}$ Burrows B, Knudson RJ, Lebowitz MD. The relationship of childhood respiratory illness to adult obstructive airway disease. Am Rev Resp Dis 1977; 115: 751-60.

${ }^{27}$ Bland JM, Holland WW, Elliott A. The development of respiratory symptoms in a cohort of Kent schoolchildren. Bull Physio-path Resp 1974; 10: 699-716.

${ }^{28}$ Holland WW, Bailey P, Bland JM. Long-term consequences of respiratory disease in infancy. $J$ Epidemiol Community Health 1978; 32: 256-9.

${ }^{29}$ Johnston IDA, Anderson HR, Lambert HP, Patel S. Respiratory morbidity and lung function after whooping cough. Lancet 1983; 2: 1104-8.

${ }^{30}$ Phelan PD. Does adult chronic obstructive lung disease really begin in childhood? $B r J$ Dis Chest $1984 ; 78$ : 1-9.

${ }^{31}$ Martin AJ, Landau LI, Phelan PD. Asthma from childhood at age 21: the patient and his disease. Br Med J 1982; 284: 380-2. 